\title{
MICORRIZA ARBUSCULAR E NUTRIÇÃO FOSFÁTICA NA TOXIDEZ DE ZINCO PARA A TREMA [Trema micrantha (L.) BLUM.] ${ }^{(1)}$
}

\author{
Cláudio Roberto Fonsêca Sousa Soares ${ }^{(2)}$, José Oswaldo Siqueira ${ }^{(3)}$, \\ Janice Guedes de Carvalho $^{(3)}$ \& Luiz Roberto Guimarães Guilherme ${ }^{(4)}$
}

\begin{abstract}
RESUMO
O objetivo deste trabalho foi avaliar os efeitos de micorriza arbuscular, do estado nutricional de $P$ da planta e de concentrações crescentes de $P$ em solução nutritiva na toxidez de Zn para Trema micrantha (L.) Blum. Em um primeiro experimento, mudas de trema foram formadas em substrato que continha doses crescentes de $\mathrm{P}\left[0,100,200\right.$ e $400 \mathrm{mg} \mathrm{dm}^{-3}$ na forma de $\left.\mathrm{Ca}\left(\mathrm{H}_{2} \mathrm{PO}_{4}\right)_{2}\right]$ e um tratamento de inoculação com Glomus etunicatum (Ge). Após crescimento por 60 dias, as mudas foram transferidas para vasos com solução nutritiva de Clark, que continha $2,75,150$ e $225 \mu \mathrm{mol} \mathrm{L}^{-1}$ de $\mathrm{Zn}$, e mantidas por mais 40 dias, quando foram colhidas e avaliadas. Os efeitos do $P$ na amenização da fitotoxidez de $\mathrm{Zn}$ foram avaliados em outro experimento, aplicando-se, simultaneamente e de forma combinada em solução, doses de $\mathrm{P}\left(0,07 ; 0,5 ; 1\right.$ e $2 \mathrm{mmol} \mathrm{L}^{-1}$ fornecido por diferentes fontes) e de $\mathrm{Zn}\left(2,75,150\right.$ e $225 \mu \mathrm{mol} \mathrm{\textrm {L } ^ { - 1 }}$ na forma de $\left.\mathrm{ZnSO}_{4} \cdot 7 \mathrm{H}_{2} \mathrm{O}\right)$, nas quais foram cultivadas mudas de trema por 40 dias. Houve acentuada inibição no crescimento e na colonização micorrízica da trema em doses elevadas de $\mathrm{Zn}$ em solução (150 e $225 \mu \mathrm{mol} \mathrm{L}^{-1}$ ). Constatou-se que a melhoria da nutrição fosfática reduziu a translocação do Zn das raízes para a parte aérea, mas isto, assim como a colonização micorrízica, não resultou em favorecimento do crescimento da planta em condições de excesso deste metal em solução. No segundo experimento, verificou-se que a elevação na concentração de $\mathbf{P}$ em solução nutritiva promoveu melhoria no estado nutricional de $\mathrm{P}$, conferindo proteção à planta do excesso de $\mathrm{Zn}$. Como a especiação química da solução indicou que a aplicação de $P$ não interferiu, de modo significativo, nas formas de Zn em solução, os resultados indicam que a ação amenizante do $\mathbf{P}$ ocorre na planta, possivelmente reduzindo a translocação do $\mathrm{Zn}$ das raízes para a parte aérea.
\end{abstract}

Termos de indexação: espécies arbóreas, fitotoxidez, fosfato, fungo micorrízico, metais pesados, tolerância.

\footnotetext{
(1) Parte da Tese de Doutorado em Solos e Nutrição de Plantas do primeiro autor, apresentada à Universidade Federal de Lavras em novembro de 2004. Trabalho financiado pelo CNPq. Recebido para publicação em fevereiro de 2005 e aprovado em julho de 2006.

(2) Assistente de Pesquisa do Departamento de Ciência do Solo, Universidade Federal de Lavras - DCS/UFLA. Caixa Postal 3037, CEP 37200-000 Lavras (MG). Bolsista Prodoc-CAPES. E-mail: crfsoares@gmail.com

(3) Professor Titular do Departamento de Ciência do Solo, UFLA. Bolsista do CNPq. E-mail: siqueira@ufla.br; janicegc@ufla.br

(4) Professor do Departamento de Ciência do Solo, UFLA. E-mail: guilherm@ufla.br
} 


\title{
SUMMARY: ARBUSCULAR MYCORRHIZA AND PHOSPHORUS NUTRITION ON ZINC TOXICITY TO TREMA [Trema micrantha (L.) BLUM.]
}

\begin{abstract}
The objectives of the present study were to evaluate the effects of arbuscular mycorrhiza, the plant $P$ nutritional status and increasing levels of $P$ in nutrient solution on $\mathrm{Zn}$ toxicity to Trema micrantha (L.) Blum. In a first experiment trema seedlings were grown in a substrate with increasing P rates [0, 100, 200 and $400 \mathrm{mg} \mathrm{dm}^{-3}$ as $\mathrm{Ca}\left(\mathrm{H}_{2} \mathrm{PO}_{4}\right)_{2}$, besides a mycorrhizal treatment inoculated with Glomus etunicatum (Ge). After 60 days of growth, the plants were transferred to pots with Clark's nutrient solution containing 2 , 75, 150 and $225 \mu \mathrm{mol} \mathrm{L} \mathrm{L}^{-1}$ of $\mathrm{Zn}$, grown for 40 days, harvested and evaluated. In a second experiment the ameliorating effects of $P$ on $\mathrm{Zn}$ phytotoxicity were also evaluated in nutrient solution containing increasing $P$ rates $\left(0.07 ; 0.5 ; 1\right.$ and $2 \mathrm{mmol} \mathrm{L}^{-1}$ supplied by different sources) and $\mathrm{Zn}\left(2,75,150\right.$ and $225 \mu \mathrm{mol} \mathrm{L} \mathrm{L}^{-1}$ as $\left.\mathrm{ZnSO}_{4} \cdot 7 \mathrm{H}_{2} \mathrm{O}\right)$ combined and simultaneously applied to the solution where trema seedlings were left to grow for 40 days. It was found that high Zn concentrations were highly inhibitory to plant growth and mycorrhizal colonization. Enhanced $P$ nutrition reduced translocation of Zn from plant roots to shoots, but such effects as well as mycorrhizal colonization had no effect on trema growth under $\mathrm{Zn}$ excess in solution. In the second experiment, higher P concentration in solution improved the plant nutritional status and protected them against the excess of $\mathrm{Zn}$. Considering that chemical speciation of the solution showed no significant effect on $\mathrm{Zn}$ forms, the results suggested that the ameliorating effects of P occur within the plant (in planta), possibly by reducing $Z n$ translocation from roots to shoots.
\end{abstract}

Index terms: woody species, phytotoxicity, phosphate, mycorrhizal fungi, heavy metals, tolerance.

\section{INTRODUÇÃO}

Os metais pesados são amplamente distribuídos nos solos e rochas onde ocorrem, normalmente, em baixas concentrações. No entanto, em áreas de mineração, transformação ou industrialização de metais, o solo pode receber elevadas concentrações destes elementos, que podem favorecer a contaminação e, conseqüentemente, acarretar problemas aos ecossistemas, o que requer intervenção antrópica para sua reabilitação (Accioly \& Siqueira, 2001). Uma estratégia para a reabilitação de áreas contaminadas com metais pesados, como $\mathrm{Cd}, \mathrm{Cu}, \mathrm{Pb}$ e Zn, é a revegetação, visando à fitoestabilização da área, especialmente com espécies arbóreas que imobilizam os metais absorvidos por mais tempo em seus tecidos, reduzindo os eventuais impactos ambientais destes elementos.

Para atingir tal objetivo, torna-se necessária a obtenção de espécies tolerantes ao excesso destes elementos (Kabata-Pendias \& Pendias, 2001) e conhecimento de mecanismos de tolerância destas espécies ao excesso de metais (Barceló \& Poschenrieder, 1992). No caso do Zn, seus teores no solo podem aumentar gradualmente em conseqüência da atividade industrial, do avanço da mineração e industrialização de materiais metálicos, uso de agroquímicos (fertilizantes e pesticidas) e deposição de resíduos no solo (Singh \& Steinnes, 1994). Quando presente em níveis tóxicos no solo, o Zn afeta o crescimento e o metabolismo das plantas (Alloway, 1993), ocasionando clorose das folhas, semelhantemente à deficiência de $\mathrm{Fe}$ (Fontes $\&$ Cox, 1998). Soares et al. (2001) verificaram que a fitotoxidez de Zn para o eucalipto está relacionada com a redução da translocação de Fe, ocasionando deficiência induzida deste elemento. $\mathrm{O} \mathrm{Zn}^{2+}$ é a forma predominante de absorção desse elemento pelas plantas, mas outras formas hidratadas, complexos e quelatos orgânicos de Zn, podem também ser absorvidas pelas raízes (Marschner, 1995).

Os mecanismos envolvidos na tolerância das plantas ao excesso de metais pesados são diversos e variados, incluindo a exsudação de substâncias quelantes, a produção intercelular de substâncias quelantes, tais como: ácidos orgânicos e fitoquelatinas e alterações na compartimentalização dos metais na célula (Shaw, 1989). Mais recentemente, estudos têm demonstrado que a inoculação de fungos micorrízicos arbusculares promove benefícios para o crescimento de plantas em solos contaminados com metais pesados, como verificado para plantas herbáceas (Gildon \& Tinker, 1983; Hetrick et al., 1994; Carneiro et al., 2001), milho (Weissenhorn et al., 1995) e mudas de plantas arbóreas (Siqueira et al., 1999). Esses estudos evidenciam o efeito protetor desta simbiose para a planta hospedeira, mas os mecanismos envolvidos nesta resposta são ainda especulativos (Gaur \& Adholeya, 2004).

Para Whitfield et al. (2004), a imobilização dos metais no micélio constitui o provável mecanismo de 
proteção das micorrizas às plantas, reduzindo a transferência do metal para a parte aérea da planta e, conseqüentemente, a fitotoxidez. Isto pode estar relacionado com a elevada capacidade de retenção de metais pesados nas estruturas dos fungos micorrízicos arbusculares, como observado por Joner et al. (2000). Além disso, é possível que as micorrizas possam contribuir para reduzir a fitotoxidez dos metais em decorrência do aumento da aquisição de P pela planta hospedeira (Silva et al., 1994; Christie et al., 2004). Isto tem relação com alguns estudos que indicam que o suprimento adequado de $\mathrm{P}$ possibilita também o aumento da retenção de metais pesados nas raízes das plantas por meio da formação de compostos insolúveis com metais, restringindo, assim, o transporte desses elementos para a parte aérea (Van Steveninck et al., 1994; Brown et al., 1995).

Van Steveninck et al. (1992) verificaram que o aumento de $\mathrm{P}$ na solução nutritiva de 0,01 para $1 \mathrm{mmol} \mathrm{L}{ }^{-1}$ reduziu a toxidez de $\mathrm{Zn}$ em diferentes clones de Lemna minor. De acordo com os autores, este efeito está relacionado com a formação de complexos de fitato de Zn nas raízes, reduzindo a translocação do metal para a parte aérea. Portanto, há evidências de que o estado nutricional de $\mathrm{P}$ da planta e as interações $\mathrm{P}$ metal podem alterar os padrões de absorção e translocação de Zn, influenciando a toxidez do metal para as plantas. Além disso, como as micorrizas melhoram a absorção de $\mathrm{P}$, torna-se necessário avaliar os efeitos e interações destes fatores na amenização da fitotoxidez de Zn.

Neste estudo, avaliou-se a influência da micorrização, do estado nutricional de $\mathrm{P}$ e de concentrações crescentes de $\mathrm{P}$ em solução nutritiva na toxidez de Zn para mudas de Trema micrantha, a qual foi selecionada por ser uma espécie pioneira de grande importância em programas de revegetação de áreas degradadas nos trópicos e pela sua sensibilidade ao excesso de metais pesados (Marques et al., 2000), e por tal espécie responder positivamente à inoculação com fungos micorrízicos e a doses de P (Paron et al., 1997).

\section{MATERIAL E MÉTODOS}

Este estudo constou de dois experimentos realizados com mudas de Trema micrantha(L.) Blum., desenvolvidos no laboratório de Microbiologia do Solo da Universidade Federal de Lavras, no período de abril de 2002 a julho de 2003. Em um primeiro experimento, avaliaram-se os efeitos de micorriza arbuscular (MA) e da condição nutricional de $\mathrm{P}$ da planta na toxidez de Zn. O experimento foi realizado em casa de vegetação, utilizando mudas de trema obtidas após tratamento das sementes com $\mathrm{H}_{2} \mathrm{SO}_{4}$ concentrado, durante 20 min, e lavadas em água corrente de modo a facilitar a germinação. Em seguida, as sementes foram colocadas em bandejas com capacidade de $6 \mathrm{dm}^{3}$ que continham substrato composto de $40 \%$ de um Latossolo Vermelho-Amarelo, $40 \%$ de areia e $20 \%$ de vermiculita (base volume), o qual foi autoclavado a $120^{\circ} \mathrm{C}$ durante 60 min (Quadro 1). O substrato de cada bandeja foi fertilizado com solução de $\mathrm{Ca}\left(\mathrm{H}_{2} \mathrm{PO}_{4}\right)_{2} \cdot \mathrm{H}_{2} \mathrm{O}$ p.a. de modo a fornecer $0,100,200 \mathrm{e}$ $400 \mathrm{mg} \mathrm{dm}^{-3}$ de $\mathrm{P}$ com vistas em obter plantas com diferente condição nutricional de $\mathrm{P}$, as quais foram posteriormente transferidas para solução nutritiva que continha doses de Zn, estabelecidas com base em outros estudos deste laboratório.

Além das doses de $\mathrm{P}$, obteve-se um tratamento de inoculação com fungos micorrízicos arbusculares e sem adição de $\mathrm{P}$, obtido pela aplicação de suspensão de esporos de Glomus etunicatum ao substrato de modo a atingir densidade de cerca de três esporos $\mathrm{mL}^{-1}$ de solo. Os esporos foram extraídos de vasos de cultivo mantidos na coleção de fungos micorrízicos arbusculares deste laboratório, por meio do peneiramento via úmida e centrifugação em solução de sacarose (Gerdemann \& Nicolson, 1963). Em cada bandeja, foram colocadas cerca de 500 sementes, as quais, após a germinação, foram cultivadas por 60 dias nos respectivos tratamentos. Após esse período, coletaram-se 10 plantas de cada bandeja para análise dos teores de nutrientes, para verificar os efeitos das doses de $\mathrm{P}$, obtendo-se mudas com teores médios de 2,1 a $4,2 \mathrm{~g} \mathrm{~kg}^{-1}$ de P. Também foram coletadas amostras de raízes frescas (2 g) do tratamento micorrízico, para avaliação do grau de colonização das mudas a serem transplantadas para as diferentes concentrações de Zn. Para isto, as raízes foram clarificadas e coloridas com azul de tripan (Koske \& Gemma, 1989) e avaliadas pelo método das intersecções em placas reticuladas (Giovannetti \& Mosse, 1980). Nesta etapa do estudo, as mudas apresentaram taxa média de colonização, de $48 \pm 9 \%$.

Quadro 1. Características químicas do substrato (após autoclavagem) utilizado para produção de mudas de trema e aplicação dos tratamentos

\begin{tabular}{lc}
\hline Características & Resultados \\
\hline $\mathrm{pH}\left(\mathrm{H}_{2} \mathrm{O}\right)$ & 6,1 \\
$\mathrm{P}\left(\mathrm{mg} \mathrm{dm}^{-3}\right)$ & 12,8 \\
$\mathrm{~K}\left(\mathrm{cmol}_{\mathrm{c} \mathrm{dm}} \mathrm{dm}^{-3}\right)$ & 0,2 \\
$\mathrm{Ca}^{2+}\left(\mathrm{cmol}_{\mathrm{c}} \mathrm{dm}^{-3}\right)$ & 1,7 \\
$\mathrm{Mg}^{2+}\left(\mathrm{cmol}_{\mathrm{c}} \mathrm{dm}^{-3}\right)$ & 1,1 \\
$\mathrm{Al}^{3+}\left(\mathrm{cmol}_{\mathrm{c}} \mathrm{dm}^{-3}\right)$ & 0,1 \\
$\mathrm{H}+{\mathrm{Al}\left(\mathrm{cmol}_{\mathrm{c}} \mathrm{dm}^{-3}\right)}_{\mathrm{SB}\left(\mathrm{cmol}_{\mathrm{c}} \mathrm{dm}^{-3}\right)}^{1,3}$ \\
$\left.\mathrm{t} \mathrm{(cmol} \mathrm{dm}^{-3}\right)$ & 3,0 \\
$\mathrm{~T}\left(\mathrm{cmol} \mathrm{dm}^{-3}\right)$ & 3,1 \\
$\mathrm{~V}(\%)$ & 4,3 \\
$\mathrm{~m}(\%)$ & 69,6 \\
& 3,0 \\
\hline
\end{tabular}


Mudas selecionadas e padronizadas foram transplantadas para bandejas hidropônicas com capacidade de $35 \mathrm{~L}$, que continham solução nutritiva de Clark (1975) com a concentração de nutrientes reduzida à metade, aeração constante e $\mathrm{pH}$ ajustado em 5,5 pela adição de $\mathrm{NaOH}$ ou $\mathrm{HCl} 0,1 \mathrm{~mol} \mathrm{~L}^{-1}$, onde foram mantidas por quatro dias. Após nova padronização quanto à altura e vigor, transplantouse uma muda por vaso de $1,7 \mathrm{~L}$ que continha a solução nutritiva de Clark com a concentração original dos nutrientes e as devidas doses de $\mathrm{Zn}$, constituindo-se na parcela experimental. Os tratamentos foram dispostos em esquema fatorial $5 \times 4$ : cinco tratamentos prévios (doses de $\mathrm{P}$ e inoculação com $G$. etunicatum) e 4 concentrações de $\mathrm{Zn}\left(2,75,150\right.$ e $225 \mu \mathrm{mol} \mathrm{L}^{-1}$ de $\mathrm{Zn}$, fornecido como $\mathrm{ZnSO}_{4} \cdot 7 \mathrm{H}_{2} \mathrm{O}$ ), no delineamento em blocos casualizados, com três repetições. As soluções foram renovadas semanalmente, aeração constante e o pH ajustado em 5,5 pela adição de $\mathrm{NaOH}$ ou $\mathrm{HCl}$ $0,1 \mathrm{~mol} \mathrm{~L}^{-1}$, sendo as plantas tratadas por 40 dias.

No segundo experimento, avaliaram-se os efeitos de doses de $\mathrm{P}$ na toxidez de $\mathrm{Zn}$ para a trema. O ensaio foi também realizado em casa de vegetação, consistindo na aplicação simultânea de doses de $\mathrm{P} \mathrm{e}$ Zn em solução nutritiva. As mudas de trema foram preparadas, conforme descrito no primeiro experimento, porém as sementes foram colocadas para germinar em bandejas que continham $6 \mathrm{dm}^{3}$ de vermiculita, onde, após emergência do primeiro par de folhas, receberam semanalmente solução nutritiva de Clark com a metade da concentração dos nutrientes. Sessenta dias após emergência, as mudas foram transplantadas para bandejas coletivas de $35 \mathrm{~L}$ que continham a mesma solução nutritiva, onde permaneceram por 20 dias, quando atingiram altura média de $30 \mathrm{~cm}$, considerada ideal para o transplante para o campo. Após padronização quanto à altura e vigor, transplantou-se uma muda por vaso de 1,7 L com a solução nutritiva de Clark com a concentração original dos demais nutrientes, ajustando-a de forma a obter diferentes concentrações de $\mathrm{P}\left(0,07 ; 0,5 ; 1\right.$ e $2 \mathrm{mmol} \mathrm{L}^{-1}$ de $\left.\mathrm{P}\right)$ e Zn $\left(2,75,150\right.$ e $225 \mu \mathrm{mol} \mathrm{L}^{-1}$ de $\left.\mathrm{Zn}\right)$, aplicadas simultaneamente e combinadas em esquema fatorial 4 x 4 (quatro doses de $\mathrm{Zn}$ e quatro de P), no delineamento em blocos casualizados, com três repetições. As doses de $\mathrm{P}$ foram definidas a partir de concentrações normalmente utilizadas em estudos com solução nutritiva, enquanto as doses de $\mathrm{Zn}$ foram definidas conforme concentrações empregadas em outros experimentos com espécies arbóreas (Paiva, 2000). As soluções de todos os tratamentos foram renovadas semanalmente, aeração constante e $\mathrm{pH}$ ajustado em 5,5 pela adição de $\mathrm{NaOH}$ ou $\mathrm{HCl} 0,1 \mathrm{~mol} \mathrm{~L}^{-1}$, sendo as plantas tratadas por 40 dias.

Em ambos os experimentos, ao final do período de exposição das plantas às doses de $\mathrm{Zn}$, realizou-se a colheita das plantas, separando-as em parte aérea e raízes. No caso do experimento 1, retiraram-se amostras de raízes para avaliação da colonização micorrízica, conforme já descrito. O restante das amostras foi colocado em estufa com circulação de ar a $65^{\circ} \mathrm{C}$ até massa constante para determinação da massa de matéria seca, moídas em moinho tipo Wiley, equipado com peneira com malha de $0,38 \mathrm{~mm}$, para digestão nítrico-perclórica e posterior análise da concentração de Zn nos tecidos por espectrofotometria de absorção atômica e de P por colorimetria por meio do método fosfo-molibdato, descrito em Malavolta et al. (1989). As quantidades acumuladas de Zn na parte aérea e raízes das plantas foram calculadas com base nas concentrações e produção de massa de matéria seca. No segundo experimento, realizou-se também a análise da especiação iônica da solução nutritiva de Clark que continha os diferentes tratamentos de P e Zn, por meio do software Visual Minteq v. 2.23. Para isto, foram utilizadas as concentrações originais dos nutrientes e metais da solução nutritiva, com vistas em determinar a atividade iônica das principais formas dos elementos e avaliar o efeito do aumento da concentração de $\mathrm{P}$ na disponibilidade de $\mathrm{Zn}$ para as plantas.

Os resultados dos dois experimentos foram submetidos à análise de variância e ao teste de médias (Scott-Knott; $\mathrm{P} \leq 0,05)$ por meio do uso do programa estatístico SISVAR (Ferreira, 2000). As equações de regressão foram determinadas pelo programa Table Curve 2D for Windows v. 5.01 (SYSTAT Software Inc), e o valor de F foi calculado e testado pelo programa F Calc32 versão 1.1. Correlações de Pearson foram realizadas por meio do programa estatístico SAEG (teste t; $\mathrm{P} \leq 0,05)$. As doses críticas de toxidez de Zn em solução (DCT) para redução em $50 \%$ da produção de matéria seca da parte aérea e raízes foram estimadas com base nas equações de regressão obtidas em resposta às concentrações dos elementos em solução.

\section{RESULTADOS E DISCUSSÃO}

As plantas de trema expostas às soluções com excesso de Zn exibiram sintomas de fitotoxidez já aos 15 dias da exposição ao metal. Ao final dos 40 dias em solução que continha Zn, observou-se que as doses de 150 e $225 \mathrm{mmol} \mathrm{L}^{-1}$ promoveram acentuada redução no crescimento das plantas (Figura 1) e clorose foliar generalizada, não sendo observado comportamento diferenciado dos tratamentos de P ou MA na evolução e na intensidade da toxidez de Zn. Houve redução na massa radicular e escurecimento das raízes, que se tornaram finas e quebradiças, à medida que aumentou a exposição ao Zn, ocasionando decréscimo de até 72 e $88 \%$ na matéria seca da parte aérea e raízes, respectivamente, independentemente dos prétratamentos aplicados na formação das mudas. Estes dados mostram que a melhoria da condição nutricional 
de P não contribuiu para a amenização do efeito tóxico do Zn. As doses críticas de toxidez de Zn em solução $\left(\mathrm{DCT}_{50}\right)$ para a matéria seca da parte aérea e raízes foram de 194 e $154 \mathrm{mmol} \mathrm{L}^{-1}$ de Zn, respectivamente, o que confirma o maior efeito tóxico deste elemento na massa radicular da trema do que na parte aérea. As $\mathrm{DCT}_{50}$ de $\mathrm{Zn}$ estimadas neste estudo foram inferiores aos valores encontrados em ensaio em condições semelhantes com Eucalyptus sp. (Soares et al., 2001), ficando evidente a maior sensibilidade da trema ao excesso de Zn.

A colonização micorrízica também foi inibida pelo excesso de $\mathrm{Zn}$, sendo esta reduzida de $54 \%$, na concentração normal de Zn, para apenas $21 \%$, na dose mais elevada em solução $\left(225 \mu \mathrm{mol} \mathrm{L}^{-1}\right)$ (Figura 2). O efeito negativo acentuou-se a partir da dose de $75 \mu \mathrm{mol} \mathrm{L}{ }^{-1}$, evidenciando a alta toxidez do Zn para o G. etunicatum, fato já relatado em outros estudos com diversas espécies (Siqueira et al., 1999; Carneiro et al., 2001). Assim como para a colonização micorrízica, verificou-se que o crescimento das plantas foi pouco influenciado até a dose de $75 \mu \mathrm{mol} \mathrm{L}{ }^{-1}$ de $\mathrm{Zn}$. No entanto, o efeito inibitório para a matéria seca e colonização micorrízica na dose de $150 \mu \mathrm{mol} \mathrm{L}^{-1} \mathrm{de} \mathrm{Zn}$ foi de 23 e $52 \%$, respectivamente, indicando que a colonização micorrízica mostrou-se mais sensível ao excesso de Zn do que o crescimento da parte aérea da trema.

Os teores de Zn na parte aérea aumentaram de 46 para $722 \mathrm{mg} \mathrm{kg}^{-1}$ com a elevação das doses desse metal em solução, representando um acréscimo de até $1.878 \%$ em relação ao controle $\left(2 \mu \mathrm{mol} \mathrm{L}^{-1}\right.$ de $\left.\mathrm{Zn}\right)$, independentemente dos pré-tratamentos aplicados (Figura 1). Houve uma relação inversa entre os teores de Zn na matéria seca da parte aérea e o crescimento das plantas, caracterizando-se o efeito tóxico do metal sobre a trema. Para avaliar melhor a relação entre a condição nutricional de $\mathrm{P}$ das plantas e os teores de Zn na matéria seca das raízes, foram selecionados, para facilidade de compreensão, os pré-tratamentos sem adição de $\mathrm{P}$, inoculação com $\mathrm{G}$. etunicatum e adição de $200 \mathrm{mg} \mathrm{dm}^{-3} \mathrm{P}$. As plantas destes tratamentos apresentaram teores de $\mathrm{P}$ na parte aérea de 2,1, 3,0 e 4,1 $\mathrm{g} \mathrm{kg}^{-1} \mathrm{P}$, respectivamente (Figura 3). Na dose mais elevada de Zn em solução $\left(225 \mu \mathrm{mol} \mathrm{L}^{-1}\right)$, os teores deste elemento nas raízes foram mais elevados nas plantas pré-inoculadas com FMA e nas précultivadas com alto $\mathrm{P}$ no substrato em comparação ao tratamento com baixo P. No entanto, este aumento na absorção de Zn das plantas com melhor condição nutricional de $\mathrm{P}$ não se refletiu nos teores deste elemento na parte aérea, uma vez que não houve comportamento diferenciado dos pré-tratamentos sobre os teores de Zn nessa área (Figura 1).

Estes resultados indicam que a nutrição fosfática das mudas de trema influenciou a translocação do Zn absorvido, situação também constatada pela razão do acúmulo proporcional de $\mathrm{Zn}$ entre a parte aérea e raízes (PA/R). O aumento das doses de $\mathrm{Zn}$ promoveu incrementos na razão de acúmulo deste elemento nas plantas com baixo suprimento de $\mathrm{P}$ e nas pré-colonizadas com FMA (Quadro 2), as quais apresentaram razão de acúmulo médio de Zn de 1,31, quando crescidas em solução com $225 \mu \mathrm{mol} \mathrm{L}^{-1} \mathrm{de} \mathrm{Zn}$, indicando haver elevada concentração desse elemento na parte aérea das plantas. Nesta mesma condição, nas mudas que apresentavam maiores teores de P na parte aérea $\left(4,1 \mathrm{~g} \mathrm{~kg}^{-1}\right)$, a razão de $\mathrm{Zn}$ foi de apenas
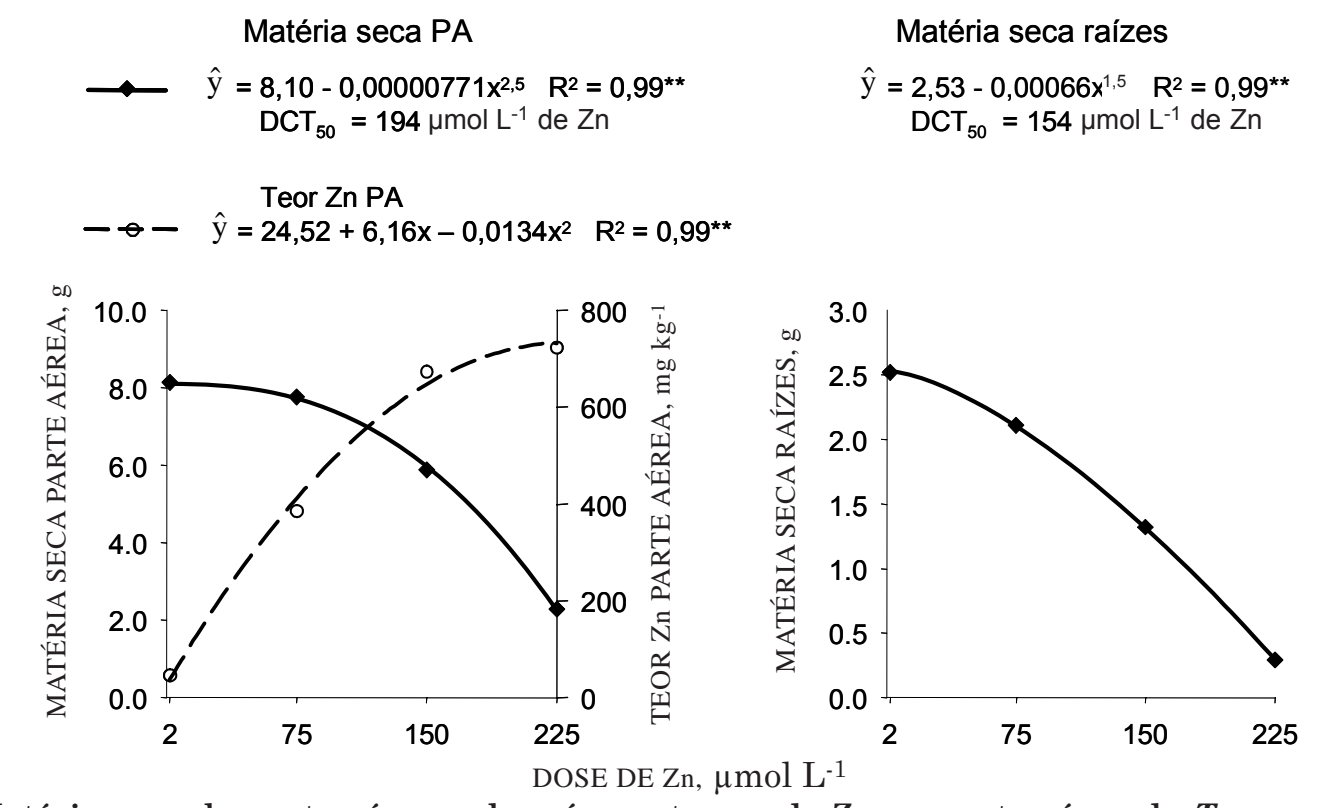

Figura 1. Matéria seca da parte aérea e de raízes e teores de Zn na parte aérea da Trema micrantha, considerando as doses de $\mathrm{Zn}$ em solução nutritiva (média dos pré-tratamentos de $\mathrm{P}$ e MA). $\mathrm{DCT}_{50}=$ concentração de $\mathrm{Zn}$ na solução que inibe em 50 \% a produção de matéria seca (** $\mathrm{P} \leq 0,01)$. 


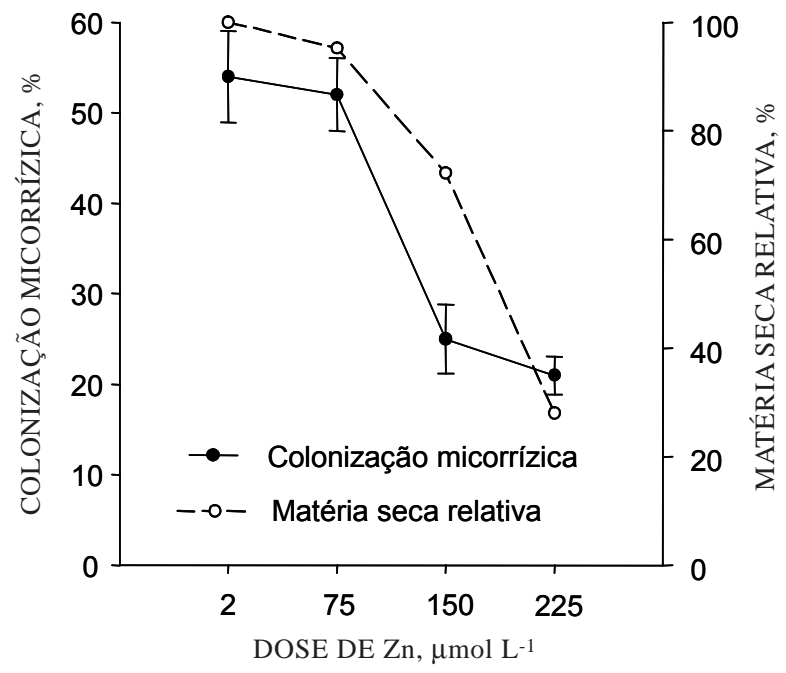

Figura 2. Colonização micorrízica e matéria seca relativa ( $\%$ do controle com $2 \mu \mathrm{mol} \mathrm{L}{ }^{-1}$ de $\mathrm{Zn}$ ) de mudas de Trema micrantha précolonizadas com G. etunicatum após 40 dias de crescimento em solução nutritiva que continha doses crescentes de $\mathrm{Zn}$. Barras verticais representam o erro-padrão da média de três repetições.

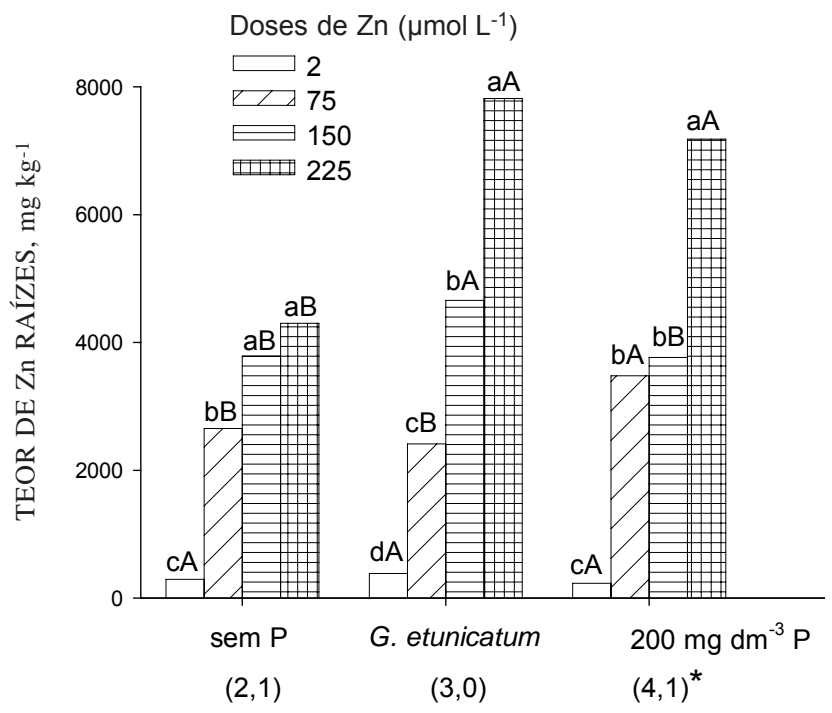

Figura 3. Relação entre a condição nutricional de $\mathbf{P}$ e os teores de Zn na matéria seca das raízes de Trema micrantha em solução nutritiva que continha doses de $\mathrm{Zn}$. Letras minúsculas comparam doses dentro da mesma condição nutricional de $\mathbf{P}$ e letras maiúsculas comparam condição nutricional de $\mathbf{P}$ dentro da mesma dose de Zn (Scott-Knott 5 \%). * Teores de P na parte aérea em $\mathrm{g} \mathrm{kg}^{-1}$

Quadro 2. Razão dos acúmulos de Zn na parte aérea e raízes (PA/R) em mudas de Trema micrantha, considerando a condição nutricional de $\mathbf{P}$ após crescimento em solução nutritiva que continha $\mathrm{Zn}$

\begin{tabular}{|c|c|c|c|}
\hline \multirow{2}{*}{ Dose de Zn } & \multicolumn{3}{|c|}{ Tratamento } \\
\hline & $2,1(\operatorname{sem} P)$ & 3,0 (G. etunicatum) & $4,1^{(1)}\left(200 \mathrm{mg} \mathrm{dm}^{-3} \mathrm{de} \mathrm{P}\right)$ \\
\hline \multicolumn{4}{|l|}{$\mu \mathrm{mol} \mathrm{L}{ }^{-1}$} \\
\hline 2 & $0,44 \mathrm{bB}$ & $0,55 \mathrm{bB}$ & $0,97 \mathrm{aA}$ \\
\hline 75 & $0,68 \mathrm{bA}$ & $0,54 \mathrm{bA}$ & $0,52 \mathrm{aA}$ \\
\hline 150 & $0,84 \mathrm{bA}$ & $0,73 \mathrm{bA}$ & $0,85 \mathrm{aA}$ \\
\hline 225 & $1,24 \mathrm{aA}$ & $1,37 \mathrm{aA}$ & $0,66 \mathrm{aB}$ \\
\hline
\end{tabular}

Letras minúsculas comparam doses de Zn dentro da mesma condição nutricional de $\mathrm{P}$ e letras maiúsculas comparam a condição nutricional de $\mathrm{P}$ dentro da mesma dose de Zn (Scott-Knott $5 \%$ ).

(1) Teores de P na parte aérea em $\mathrm{g} \mathrm{kg}^{-1}$.

0,66. Estes resultados mostram que, apesar de não haver contribuição dos tratamentos de $\mathrm{P}$ ou MA para o crescimento da trema, o aumento no suprimento de $\mathrm{P}$ propiciou maior retenção do Zn nas raízes, restringindo sua translocação para a parte aérea. Tal resultado corrobora outros estudos em que o suprimento adequado de $\mathrm{P}$ favoreceu a retenção de metais pesados nas raízes (Van Steveninck et al., 1994; Brown et al., 1995). Segundo esses autores isto estaria relacionado com a formação de compostos insolúveis capazes de reduzir o transporte do Zn para a parte aérea.
Os teores de $\mathrm{P}$ na parte aérea da trema aumentaram de 1,0 para $2,2 \mathrm{~g} \mathrm{~kg}^{-1} \mathrm{P}$, com a elevação das doses de $\mathrm{Zn}$ em solução, exibindo resposta quadrática $\left(\mathrm{Y}=1,01-0,00023 \mathrm{X}+0,000024 \mathrm{X}^{2} ; \mathrm{R}^{2}=\right.$ $0,99 ; \mathrm{P} \leq 0,01)$, independentemente dos prétratamentos aplicados. Este comportamento pode estar relacionado com o efeito de concentração, uma vez que houve redução no crescimento da planta com o aumento das doses de Zn em solução (Figura 1). A reduzida influência dos pré-tratamentos nos teores de P na parte aérea das plantas pode ser atribuída à baixa concentração desse nutriente na solução nutritiva 
$\left(0,07 \mathrm{mmol} \mathrm{L}^{-1} \mathrm{de} \mathrm{P}\right)$, fornecida durante os 40 dias de crescimento das plantas em solução que continha as doses de Zn. Segundo Barceló \& Poschenrieder (1992), a toxidez de Zn para as plantas relaciona-se com desequilíbrios da relação $\mathrm{P} / \mathrm{Zn}$ nos tecidos.

Considerando a razão da concentração P/Zn na parte aérea da trema na dose de $2 \mu \mathrm{mol} \mathrm{L} \mathrm{L}^{-1}$ de $\mathrm{Zn}$ como a ideal para as plantas, verificou-se que esta foi reduzida de 23,4 para apenas $3,0 \mathrm{mg} \mathrm{mg}^{-1}$, na dose de $225 \mu \mathrm{mol} \mathrm{L}^{-1}$ de $\mathrm{Zn}$, sem comportamento diferenciado entre os pré-tratamentos de P e FMA (dados não mostrados), ficando evidente a relação entre a toxidez de $\mathrm{Zn}$ para a trema e os desequilíbrios da relação $\mathrm{P} / \mathrm{Zn}$ na planta.

As plantas do segundo experimento mostraram sintomas semelhantes aos relatados no primeiro experimento, quando expostas às doses mais elevadas de Zn. Houve também acentuada redução no crescimento da parte aérea e raízes (Figura 4), com o aparecimento de clorose foliar e murchamento das folhas na dose mais elevada de Zn em solução $\left(225 \mu \mathrm{mol} \mathrm{L}^{-1}\right)$. Entretanto, o aumento da concentração de $\mathrm{P}$ em solução retardou o aparecimento destes sintomas, indicando um efeito protetor do $\mathrm{P}$ sobre a toxidez de $\mathrm{Zn}$ nas plantas. $\mathrm{Na}$ concentração normal de $\mathrm{Zn}$ na solução $\left(2 \mu \mathrm{mol} \mathrm{L}^{-1}\right)$, o aumento do fornecimento de $\mathrm{P}$ promoveu incrementos na produção de matéria seca da trema, evidenciando a resposta positiva da aplicação desse nutriente no crescimento dessa espécie. No entanto, o aumento das doses de Zn em solução limitou a resposta ao P. Mesmo na dose mais elevada de $\mathrm{P}$ em solução $\left(2 \mathrm{mmol} \mathrm{L}^{-1}\right)$, a toxidez do $\mathrm{Zn}$ foi evidente, limitando o crescimento das plantas (Figura 4).
Verificou-se um decréscimo médio de $50 \%$ da matéria seca da trema crescendo em $225 \mu \mathrm{mol} \mathrm{L}{ }^{-1}$ de $\mathrm{Zn}$ em relação à dose de $2 \mu \mathrm{mol} \mathrm{L}^{-1}$ de $\mathrm{Zn}$. Apesar disso, notou-se que mesmo em concentrações tóxicas de Zn, a adição de altas doses de $P$ possibilitou maior produção de matéria seca das plantas em comparação àquelas com baixo fornecimento de $\mathrm{P}$.

$\mathrm{O}$ aumento na concentração de $\mathrm{P}$ em solução de 0,07 para $2 \mathrm{mmol} \mathrm{L}^{-1}$ elevou as $\mathrm{DCT}_{50}$ estimadas para as raízes de 101 para $203 \mu \mathrm{mol} \mathrm{L}^{-1}$ de Zn (Quadro 3). Além disso, o aumento das doses de $\mathrm{P}$ também elevou as $\mathrm{DCT}_{50}$ estimadas para a parte aérea, indicando que o aumento no fornecimento de $\mathrm{P}$ à solução teve efeito

Quadro 3. Doses críticas de toxidez $\left(\mathrm{DCT}_{50}\right)^{(1)}$ de $\mathrm{Zn}$ em solução nutritiva para a matéria seca da parte aérea e raízes de Trema micrantha, considerando diferentes doses de $P$ (experimento 2)

\begin{tabular}{ccc}
\hline Dose de $\mathbf{P}$ & Parte aérea & Raiz \\
\hline mmol L $^{-1}$ & - DCT $_{50}, \mu \mathrm{mol} \mathrm{L}^{-1}$ de Zn - \\
0,07 & n.e. & 101 \\
0,5 & 194 & 135 \\
1,0 & 175 & 150 \\
2,0 & 220 & 203 \\
\hline (1) Concentração de Zn em solução que inibe em $50 \%$ a biomassa \\
das plantas. \\
n.e. = não estimado graças ao efeito não-significativo das doses \\
de Zn.
\end{tabular}
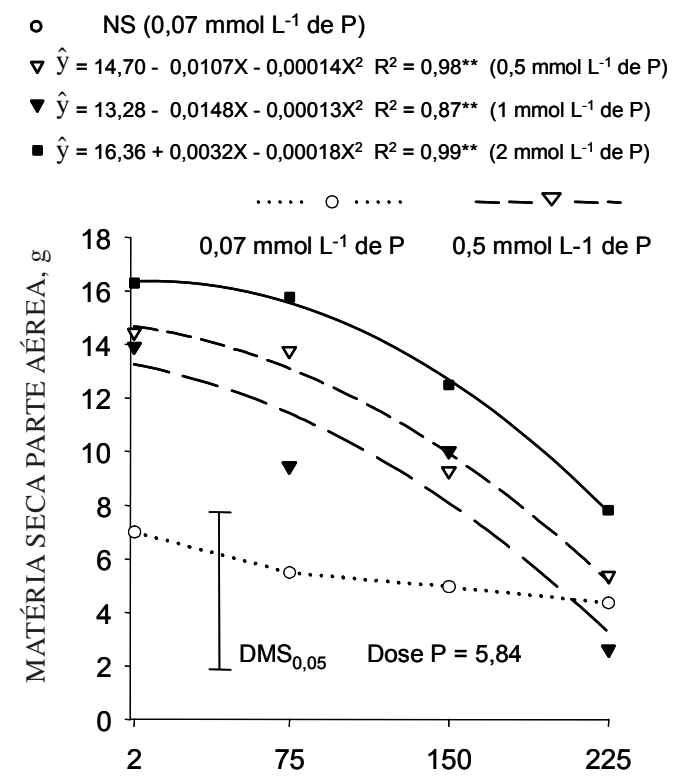

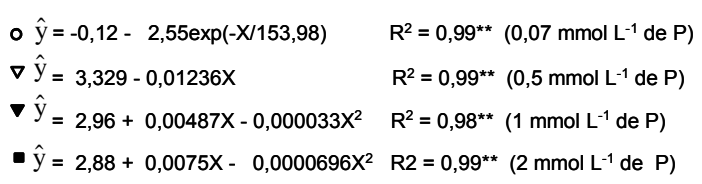

- $\hat{y}=2,88+0,0075 X-0,0000696 X^{2} \quad R 2=0,99^{* *}\left(2 \mathrm{mmol} \mathrm{L}^{-1}\right.$ de P $)$

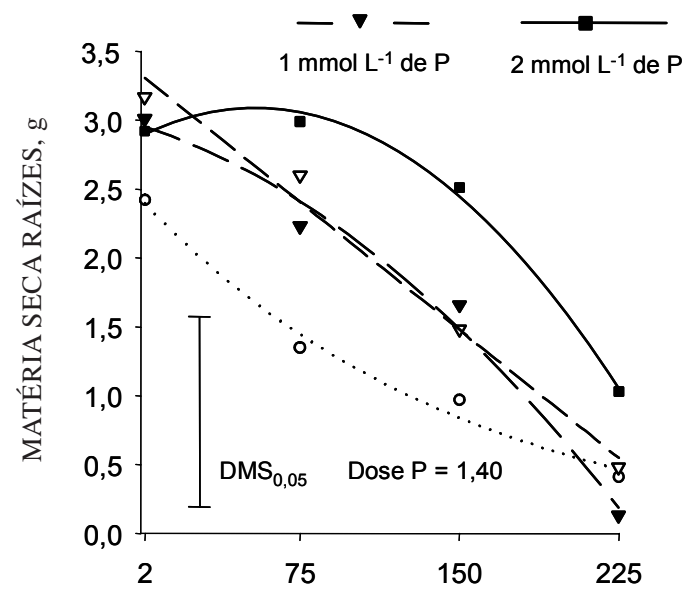

DOSE DE Zn, $\mu \mathrm{mol} \mathrm{L} \mathrm{L}^{-1}$

Figura 4. Matéria seca da parte aérea e raízes de mudas de Trema micrantha em resposta ao aumento de doses de Zn e aplicação de $\mathrm{P}$ em solução nutritiva (** $\mathrm{P}=0,01$; NS = não-significativo). 
amenizante da fitotoxidez de $\mathrm{Zn}$ para a trema. As doses críticas de toxidez de Zn em solução estimadas para as raízes foram inferiores àquelas obtidas para a parte aérea, evidenciando a maior susceptibilidade das raízes ao excesso de $\mathrm{Zn}$, como verificado no experimento 1.

Os teores de Zn nas raízes aumentaram de 756 para $5.040 \mathrm{mg} \mathrm{kg}^{-1}$ de acordo com o aumento das doses de $\mathrm{Zn}$ em solução, exibindo resposta exponencial $\left[\mathrm{Y}=542,64+1315 \exp (\mathrm{X} / 155,6), \mathrm{R}^{2}=\right.$ $0,97 ; \mathrm{P} \leq 0,01]$. Neste caso, não foi verificado comportamento diferenciado entre as doses de $\mathrm{P}$ aplicadas e isto pode ser devido à baixa influência da adição de $\mathrm{P}$ sobre a percentagem das principais formas de $\mathrm{Zn}$ disponíveis para a planta $\left(\mathrm{Zn}^{2+} \mathrm{e}\right.$ ZnEDTA $^{2-}$ ), conforme revelou o estudo de especiação iônica (Quadro 4). Estas formas representaram mais de $90 \%$ da concentração total de Zn em solução, enquanto outras consideradas menos disponíveis para a absorção, como o $\mathrm{ZnHPO}_{4}$ (Alloway, 1993), não ultrapassaram os $2 \%$. Deve-se salientar que a formação de ZnEDTA ${ }^{2-}$ está relacionada com a adição de EDTA para fornecimento de Fe à solução nutritiva. Portanto, o aumento das doses de $\mathrm{P}$ não interfere de modo relevante na disponibilidade de Zn na solução nas condições do presente estudo, indicando que seus efeitos sobre a atenuação da fitotoxidez de Zn são de ordem fisiológica e nutricional na planta.

Ao contrário do que foi observado para as raízes, notou-se que os teores de Zn na parte aérea foram influenciados pelo aumento das doses de $\mathrm{P}$ em solução (Figura 5). Considerando a dose de $150 \mu \mathrm{mol} \mathrm{L}{ }^{-1}$ de $\mathrm{Zn}$, verificou-se que a adição de $1 \mathrm{mmol} \mathrm{L}^{-1}$ de $\mathrm{P}$ à solução foi capaz de reduzir em mais de $26 \%$ os teores de Zn na matéria seca da parte aérea, em comparação às demais doses de $\mathrm{P}$ aplicadas (Figura 5), evidenciando o antagonismo na absorção entre estes dois elementos (Marschner, 1995). Entretanto, tal comportamento não foi constatado, quando se adicionaram $2 \mathrm{mmol} \mathrm{L}^{-1}$ de $\mathrm{P}$, que proporcionaram maior crescimento das plantas e, conseqüentemente, maior capacidade de absorção de Zn, em comparação aos tratamentos com baixo fornecimento de P. Desta forma, estudos adicionais em âmbito molecular, celular e físiológico são necessários, para delinear os mecanismos pelos quais a nutrição fosfática interage com a absorção e efeitos do excesso de Zn na planta. Deve-se considerar que um maior suplemento de $\mathrm{P}$ pode aumentar a síntese de fitatos, os quais são conhecidos por exibir elevada capacidade de complexar Zn no córtex das raízes (Van Steveninck et al., 1994), podendo atuar diretamente na amenização da toxidez de Zn.

Quadro 4. Atividade iônica e percentagem das principais formas de Zn e P adicionados simultaneamente à solução nutritiva (experimento 2)

\begin{tabular}{|c|c|c|c|c|}
\hline \multirow[b]{2}{*}{ Dose em solução } & \multicolumn{4}{|c|}{ Atividade de íons } \\
\hline & $\mathrm{Zn}^{2+}$ & $\operatorname{ZnEDTA}{ }^{2-}$ & $\mathrm{ZnHPO}_{4}$ & $\mathrm{H}_{2} \mathrm{PO}_{4}^{-}$ \\
\hline $2 \mu \mathrm{mol} \mathrm{L}^{-1} \mathrm{de} \mathrm{Zn}$ & 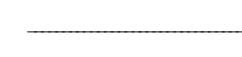 & $-\mu$ & - & - \\
\hline $\begin{array}{l}+0,07 \mathrm{mmol} \mathrm{L}^{-1} \text { de } \mathrm{P} \\
+0,5 \mathrm{~m} \mathrm{~mol} \mathrm{~L}^{-1} \text { de } \mathrm{P} \\
+1,0 \mathrm{~m} \mathrm{~mol} \mathrm{~L}^{-1} \text { de } \mathrm{P} \\
+2,0 \mathrm{mmol} \mathrm{L}^{-1} \text { de } \mathrm{P}\end{array}$ & $\begin{array}{l}0,0014(0,1)^{(1)} \\
0,0014(0,1) \\
0,0013(0,1) \\
0,0013(0,1)\end{array}$ & $\begin{array}{l}1,26(99) \\
1,26(99) \\
1,27(99) \\
1,29(99)\end{array}$ & $\begin{array}{l}<0,1(--) \\
<0,1(--) \\
<0,1(--) \\
<0,1(--)\end{array}$ & $\begin{array}{r}52(92) \\
408(92) \\
601(93) \\
1123(94)\end{array}$ \\
\hline $\begin{array}{l}\quad 75 \mu \mathbf{m o l ~ L} \\
+ \\
+0,07 \mathrm{mmol} \mathrm{L}^{-1} \text { de } \mathrm{P} \\
+0,5 \mathrm{mmol} \mathrm{L}^{-1} \text { de } \mathrm{P} \\
+1,0 \mathrm{mmol} \mathrm{L}^{-1} \text { de } \mathrm{P} \\
+2,0 \mathrm{~m} \mathrm{~mol} \mathrm{~L}^{-1} \text { de } \mathrm{P}\end{array}$ & $\begin{array}{l}24,2(48) \\
23,9(48) \\
24,0(48) \\
23,9(47)\end{array}$ & $\begin{array}{l}23,6(48) \\
23,6(48) \\
23,8(48) \\
24,2(48)\end{array}$ & $\begin{aligned}<0,1 & (--) \\
0,4 & (0,5) \\
0,6 & (0,8) \\
1,1 & (1,5)\end{aligned}$ & $\begin{aligned} 51 & (92) \\
407 & (92) \\
604 & (93) \\
1126 & (94)\end{aligned}$ \\
\hline $\begin{array}{l}150 \mu \mathbf{m o l ~ L}^{-1} \text { de } \mathbf{Z n} \\
+0,07 \mathrm{mmol} \mathrm{L}^{-1} \text { de } \mathrm{P} \\
+0,5 \mathrm{mmol} \mathrm{L}^{-1} \text { de } \mathrm{P} \\
+1,0 \mathrm{mmol} \mathrm{L}^{-1} \text { de } \mathrm{P} \\
+2,0 \mathrm{mmol} \mathrm{L}^{-1} \text { de } \mathrm{P}\end{array}$ & $\begin{array}{l}69,5(71) \\
68,8(70) \\
68,9(70) \\
68,7(68)\end{array}$ & $\begin{array}{l}23,5(25) \\
23,5(25) \\
23,7(25) \\
24,1(25)\end{array}$ & $\begin{array}{l}0,2(0,1) \\
1,2(0,8) \\
1,7(1,1) \\
3,2(2,0)\end{array}$ & $\begin{aligned} 51 & (92) \\
406 & (92) \\
607 & (93) \\
1128 & (94)\end{aligned}$ \\
\hline $\begin{array}{l}225 \mu \text { mol L }^{-1} \text { de } \mathbf{Z n} \\
+0,07 \mathrm{mmol} \mathrm{L}^{-1} \text { de } \mathrm{P} \\
+0,5 \mathrm{mmol} \mathrm{L}^{-1} \text { de } \mathrm{P} \\
+1,0 \mathrm{mmol} \mathrm{L}^{-1} \text { de } \mathrm{P} \\
+2,0 \mathrm{mmol} \mathrm{L}^{-1} \text { de } \mathrm{P}\end{array}$ & $\begin{array}{r}114(78) \\
113(77) \\
113(77) \\
76(70)\end{array}$ & $\begin{array}{l}23,5(16) \\
23,4(16) \\
23,6(17) \\
24,0(23)\end{array}$ & $\begin{array}{l}0,2(0,1) \\
1,9(0,8) \\
2,9(1,3) \\
3,5(2,0)\end{array}$ & $\begin{aligned} 51 & (92) \\
406 & (92) \\
610 & (93) \\
1106 & (94)\end{aligned}$ \\
\hline
\end{tabular}

(1) Valores entre parênteses representam a percentagem da concentração total do componente. 
$\mathrm{O}$ aumento das doses de $\mathrm{Zn}$ e a aplicação de $\mathrm{P}$ à solução exerceram efeitos significativos sobre os teores de $\mathrm{P}$ na matéria seca da parte aérea da trema (Figura 6). Verifica-se que, de maneira geral, o aumento das doses de $\mathrm{Zn}$ reduziu os teores de $\mathrm{P}$ na matéria seca da parte aérea. Isto pode estar relacionado com o efeito de metais na absorção e translocação deste nutriente na planta. Miranda \& Rowell (1989) verificaram que o excesso de Al reduziu a translocação de $\mathrm{P}$ no trigo. Mesmo ocorrendo a redução dos teores de $\mathrm{P}$ na trema, a elevação do fornecimento deste nutriente em solução propiciou mudas com melhor condição nutricional de $\mathrm{P}$, mesmo em concentrações tóxicas de Zn. A contribuição da
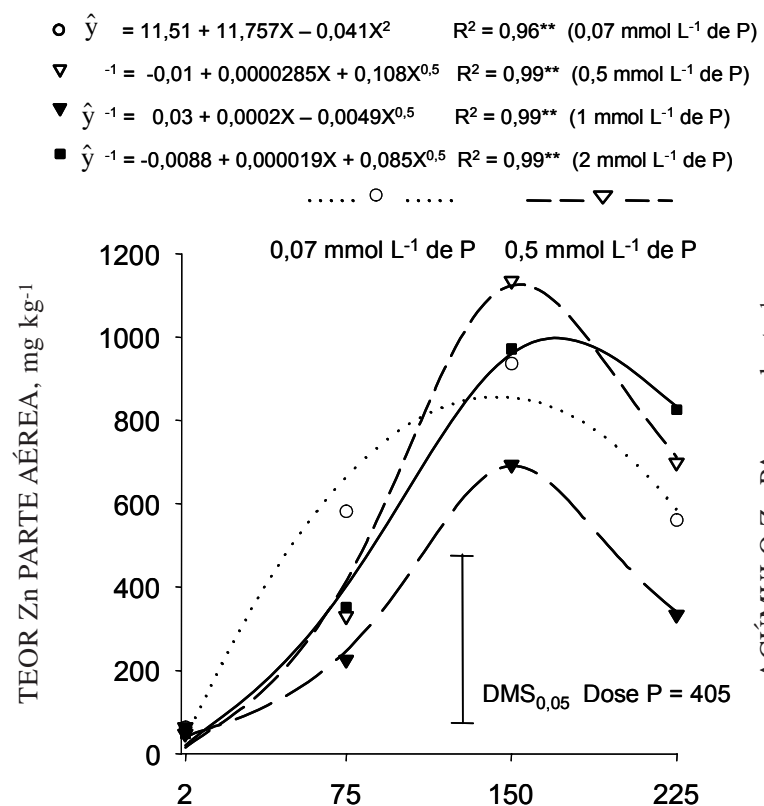
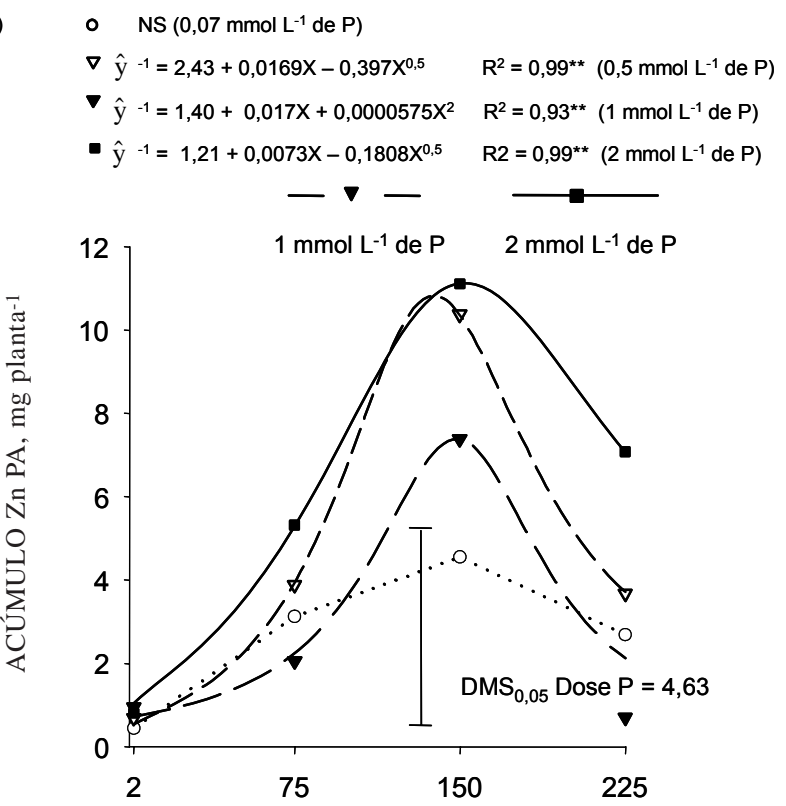

DOSE DE Zn, $\mu \mathrm{mol} \mathrm{L}{ }^{-1}$

Figura 5. Teor e acúmulo de Zn na parte aérea de mudas de Trema micrantha em resposta ao aumento de doses de Zn e aplicação de $\mathrm{P}$ em solução nutritiva (** $\mathrm{P} \leq 0,01$; NS = não-significativo).

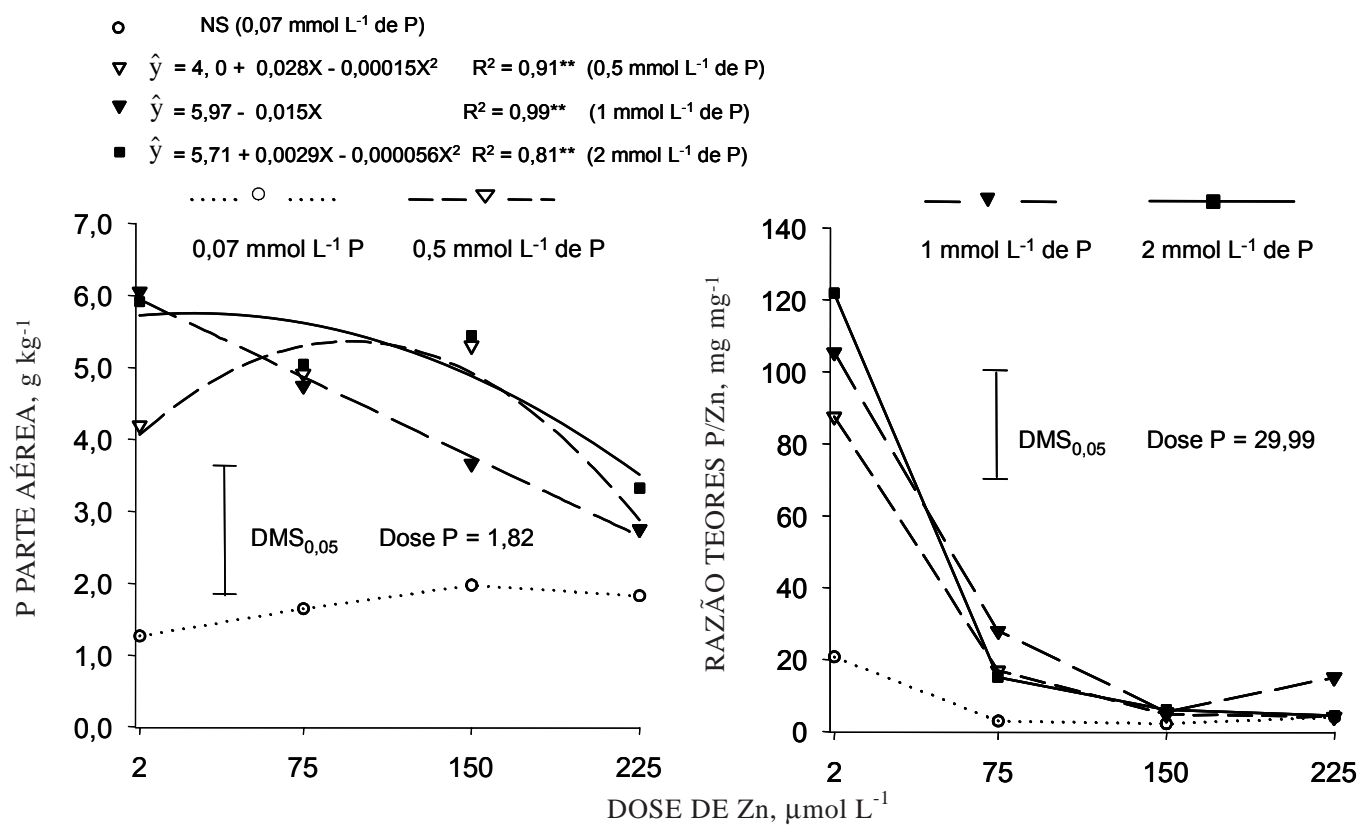

Figura 6. Teor de $\mathrm{P}$ e razão da concentração $\mathrm{P} / \mathrm{Zn}$ na parte aérea de mudas de Trema micrantha em resposta ao aumento de doses de Zn e aplicação de $\mathrm{P}$ em solução nutritiva (** $\mathrm{P} \leq 0,01 ; * \mathrm{P} \leq 0,05$; NS = não-significativo). 
condição nutricional de $\mathrm{P}$ pode ser constatada por meio da correlação positiva entre os teores de $\mathrm{P}$ na planta e a produção de matéria seca da parte aérea $\left(\mathrm{r}=0,83^{* * *}\right)$ e raízes $\left(r=0,69^{* *}\right)$. Assim, a melhoria da condição nutricional de $\mathrm{P}$ pelo aumento do fornecimento deste nutriente favorece o crescimento da trema mesmo em condições de excesso de Zn em solução. Apesar disso, pôde-se notar que o aumento da concentração de $\mathrm{P}$ garantiu relação $\mathrm{P} / \mathrm{Zn}$ mais favorável apenas na dose mais baixa de $\mathrm{Zn}\left(2 \mu \mathrm{mol} \mathrm{L}{ }^{-1}\right)$, sendo esta reduzida substancialmente com a elevação das doses do metal em solução (Figura 6), assim como observado para o experimento 1. A partir da dose de $75 \mu \mathrm{mol} \mathrm{L}^{-1}$ de $\mathrm{Zn}$, não houve efeito da adição de $\mathrm{P}$ na razão da concentração $\mathrm{P} / \mathrm{Zn}$ na parte aérea, indicando que a toxidez de Zn está relacionada com o desequilíbrio da relação P/Zn na planta.

\section{CONCLUSÕES}

1. O excesso de $\mathrm{Zn}$ foi inibitório para o crescimento e colonização micorrízica da trema, sendo a colonização mais sensível ao excesso de Zn em solução do que o crescimento da planta.

2. Os pré-tratamentos com $\mathrm{P}$ e a inoculação com o fungo micorrízico Glomus etunicatum não exerceram efeito protetor da toxidez de Zn para a trema, mas plantas com melhor suprimento de $\mathrm{P}$ tiveram menor translocação de $\mathrm{Zn}$ das raízes para a parte aérea.

3. O aumento da concentração de $\mathrm{P}$ aplicado simultaneamente com o $\mathrm{Zn}$ em solução nutritiva melhorou o estado nutricional de $\mathrm{P}$ e conferiu proteção da toxidez de Zn para a trema. No entanto, a adição de P não teve efeito sobre a relação $\mathrm{P} / \mathrm{Zn}$ na planta em condições de excesso de Zn em solução.

\section{LITERATURA CITADA}

ACCIOLY, A.M.A. \& SIQUEIRA, J.O. Contaminação química e biorremediação do solo. In: NOVAIS, R.F.A.; ALVAREZ V., V.H. \& SCHAEFER, C.E., eds.. Tópicos em ciência do solo. Viçosa, MG, Sociedade Brasileira de Ciência do Solo, 2000. v.1, p.299-351.

ALLOWAY, B.J. Heavy metals in soils. New York, John Wiley, 1993. 339p.

BARCELÓ, J. \& POSCHENRIEDER, Ch. Respuestas de las plantas a la contaminacion por metales pesados. Suelo Planta, 2:345-361, 1992.

BROWN, S.L.; CHANEY, R.L.; ANGLE, J.S. \& BAKER, A.J.M. Zinc and cadmium uptake by hyperaccumulator Thlaspi coerulescens grown in nutrient solution. Soil Sci. Soc. Am. J., 59:125-133, 1995.
CARNEIRO, M.A.C.; SIQUEIRA, J.O. \& MOREIRA, F.M.S. Estabelecimento de plantas herbáceas em solo com contaminação de metais pesados e inoculação de fungos micorrízicos arbusculares. Pesq. Agropec. Bras., 36:14431452,2001

CHRISTIE, P.; LI, X. \& CHEN, B. Arbuscular mycorrhiza can depress translocation of zinc to shoots of host plants in soils moderately polluted with zinc. Plant Soil, 261:209. 217,2004

CLARK, R.B. Characterization of phosphates in intact maize roots. J. Agric. Food Chem., 23:458-460, 1975.

FERREIRA, D.F. Análise estatística por meio do SISVAR para Windows versão 4.0. In: REUNIÃO BRASILEIRA DA SOCIEDADE INTERNACIONAL DE BIOMETRIA, 45., São Carlos, 2000. Programas e Resumos. São Carlos, Universidade Federal de São Carlos, 2000. p.235.

FONTES, R.L.F. \& COX, R. Iron deficiency and zinc toxicity in soybean grown in nutrient solution with different levels of sulfur. J. Plant Nutr., 21:1715-1722, 1998.

GAUR, A. \& ADHOLEYA, A. Prospects of arbuscular mycorrhizal fungi in phytoremediation of heavy metal contaminated soils. Curr. Sci. India, 86:528-534, 2004.

GERDEMANN, J.B. \& NICOLSON, T.H. Spores of mycorrhizal Endogone species extracted from soil by wet sieving and decanting. Trans. Brit. Mycol. Soc., 46:235-246, 1963.

GILDON, A. \& TINKER, P.B. Interactions of vesiculararbuscular mycorrhizal infection and heavy metals in plants. I. The effects of heavy metals on the development of vesicular-arbuscular mycorrhizas. New Phytol., 95:247$261,1983$.

GIOVANNETTI, M. \& MOSSE, B. An evaluation of techniques to measure vesicular-arbuscular infection in roots. New Phytol., 84:489-500, 1980.

HETRICK, B.A.D.; WILSON, G.W.T. \& FIGGE, D.A.H. The influence of mycorrhizal symbiosis and fertilizer amendments on establishment of vegetation in heavy metal mine spoil. Environ. Pollut., 86:171-179, 1994.

JONER, E.J.; BRIONES, R. \& LEYVAL, C. Metal-binding capacity of arbuscular mycorrhizal mycelium. Plant Soil, 226:227-234, 2000.

KABATA-PENDIAS, A. \& PENDIAS, H. Trace elements in soils and plants. Boca Raton, CRC Press, 2001. 413p.

KOSKE, R.E. \& GEMMA, J.N. A modified procedure for staining roots to detect VA mycorrhizas. Mycol. Res., 92:486-488, 1989.

MALAVOLTA, E.; VITTI, G.C. \& OLIVEIRA, S.A. Avaliação do estado nutricional das plantas: princípios e aplicações. Piracicaba, POTAFÓS, 1989. 201p.

MARQUES, T.C.L.L.S.M.; MOREIRA, F.M.S. \& SIQUEIRA, J.O. Crescimento e absorção de metais em mudas de espécies arbóreas em solo contaminado com metais pesados. Pesq. Agropec. Bras., 35:121-132, 2000.

MARSCHNER, H. Mineral nutrition of higher plants. 2.ed. San Diego, Academic Press, 1995. 902p. 
MIRANDA, L.N. \& ROWELL, D.L. Aluminium-phosphate interactions in wheat. New Phytol., 113:7-12, 1989.

PAIVA, H.N. Toxidez de $\mathrm{Cd}, \mathrm{Ni}, \mathrm{Pb}$ e $\mathrm{Zn}$ em mudas de cedro (Cedrella fissilis Vell.) e ipê-roxo (Tabebuia impetiginosa (Mart.) Standl.). Lavras, Universidade Federal de Lavras, 2000. 283p. (Tese de Doutorado).

PARON, M.E.; SIQUEIRA, J.O. \& CURI, N. Fungos micorrízicos, fósforo e nitrogênio no crescimento inicial da trema e do fededoso. R. Bras. Ci. Solo, 21:567-574, 1997.

SHAW, A.J. Heavy metal tolerance in plants: evolutionary aspects. New York, CRC Press, 1989. 355p.

SILVA, L.H.B.; MIRANDA, J.C.C. \& MIRANDA, L.N. Efeito da micorriza vesiculo-arbuscular no crescimento de variedades de trigo sensível e tolerante ao alumínio, em solo de cerrado. R. Bras. Ci. Solo, 18:407-414, 1994.

SINGH, B.R. \& STEINNES, E. Soil and water contamination by heavy metal. In: LAL, R. \& STEWART, B.A., eds. Soil process and water quality. Boca Raton, Lewis Publishers, 1994. p.233-271.

SIQUEIRA, J.O.; POUYÚ, E. \& MOREIRA, F.M.S. Micorrizas arbusculares no crescimento pós-transplantio de mudas de árvores em solo com excesso de metais pesados. R. Bras. Ci. Solo, 23:569-580, 1999.
SOARES, C.R.F.S.; GRAZZIOTTI, P.H.; SIQUEIRA, J.O.; CARVALHO, J.G. \& MOREIRA, F.M.S. Toxidez de zinco no crescimento e nutrição de Eucalyptus maculata e Eucalyptus urophylla em solução nutritiva. Pesq. Agropec. Bras., 36:339-348, 2001.

van STEVENINCK, R.F.M.; van STEVENINCK, M.E. \& FERNANDO, D.R. Heavy-metal (Zn, Cd) tolerance in selected clones of duck weed (Lemna minor). Plant Soil, 146:271-280, 1992.

van STEVENINCK, R.F.M.; BABARE, A.; FERNANDO, D.R. \& van STEVENINCK, M.E. The binding of zinc, but not cadmium, by phytic acid in roots of crop plants. Plant Soil, 167:157-164, 1994.

WEISSENHORN, I.; LEYVAL, C.; BELGY, G. \& BERTHELIN, J. Arbuscular mycorrhizal contribution to heavy metal uptake by maize (Zea mays L.) in pot culture with contaminated soil. Mycorrhiza, 5:245-251, 1995.

WHITFIELD, L.; RICHARDS, A.J. \& RIMMER, D.L. Effects of mycorrhizal colonisation on Thymus polytrichus from heavy-metal-contaminated sites in Northern England. Mycorrhiza, 14:47-54, 2004. 